\title{
Efficiency of a self-administered outpatient parenteral antimicrobial therapy (s-opat) for infective endocarditis within the context of a shortened hospital admission based on hospital at home program
}

\author{
Marcos Pajarón, Marta Lisa, Manuel F Fernández-Miera, Juan C Dueñas, I \\ Allende, Ana M Arnaiz, Pedro Sanroma-Mendizábal, José R de Berrazueta \& \\ María C Fariñas
}

To cite this article: Marcos Pajarón, Marta Lisa, Manuel F Fernández-Miera, Juan C Dueñas, I Allende, Ana M Arnaiz, Pedro Sanroma-Mendizábal, José R de Berrazueta \& María C Fariñas (2017): Efficiency of a self-administered outpatient parenteral antimicrobial therapy (s-opat) for infective endocarditis within the context of a shortened hospital admission based on hospital at home program, Hospital Practice, DOI: 10.1080/21548331.2017.1398588

To link to this article: http://dx.doi.org/10.1080/21548331.2017.1398588

Accepted author version posted online: 01 Nov 2017.

Submit your article to this journal ๘

View related articles ¿

View Crossmark data [ 
Publisher: Taylor \& Francis

Journal: Hospital Practice

DOI: $10.1080 / 21548331.2017 .1398588$

Efficiency of a self-administered outpatient parenteral antimicrobial therapy (s-opat) for infective endocarditis within the context of a shortened hospital admission based on hospital at home program

Marcos Pajarón, Marta Lisa, Manuel F Fernández-Miera, Juan C Dueñas, I Allende, Ana M Arnaiz, Pedro Sanroma-Mendizábal, José R de Berrazueta, María C Fariñas; The Hospital Marqués de Valdecilla Endocarditis Study Group. *

* Study group: Carlos Armiñanzas, Ana M Arnaiz, Manuel Cobo-Belaustegui, Jose R de Berrazueta, Maria C Fariñas, Jesús Agüero, Marta Fernández-Sampedro, Claudia GonzálezRico, Manuel Gutiérrez-Cuadra, Francisco Gutierrez-Diez, Rubén G Izquierdo, Marcos Pajarón, Ramon Teira, and Jose Zarauza

Author affiliations

Marcos Pajarón Guerrero, MD, PhD, Domiciliary Hospitalisation Unit, Hospital Universitario Marqués de Valdecilla, Santander, Spain, e-mail: mpajaron@humv.es

Marta Lisa, MD, Internal Medicine Unit, Hospital Universitario Marqués de Valdecilla, Santander, Spain, e-mail: m.lisagracia@gmail.com

Manuel Francisco Fernández Miera, MD, Domiciliary Hospitalisation Unit, Hospital Universitario Marqués de Valdecilla, Santander, Spain, e-mail: hadfmm@humv.es Juan Carlos Dueñas Puebla MD, Financial Management, Hospital Universitario Marqués de Valdecilla, Santander, Spain, e-mail: juancarlos@humv.es

Iciar Allende Mancisidor, MD, Primary Care and Community Medicine. Santa Cruz de Bezana Santander, Spain, e-mail: iciar74@gmail.com

Ana M Arnaiz, MD, PhD, Infectious Disease Unit, Hospital Universitario Marqués de Valdecilla, Santander, Spain, e-mail: anarnaiz@humv.es

Pedro Sanroma, MD, Domiciliary Hospitalisation Unit, Hospital Universitario Marqués de Valdecilla, Santander, Spain, e-mail: hadsmp@humv.es 
Jose Ramon De Berrazueta MD, PhD, Service of Cardiology, Hospital Universitario Marqués de Valdecilla, University of Cantabria, Santander, Spain, e-mail: carbfi@humv.es

María C Fariñas, MD, PhD, Infectious Disease Unit, Hospital Universitario Marqués de Valdecilla, University of Cantabria, Santander, Spain, e-mail: mcfarinas@humv.es

\section{Corresponding autor}

Marta Lisa, MD, Internal Medicine Unit, Hospital Universitario Marqués de Valdecilla, Avda. Valdecilla s/n, 39008. Santander, Spain.

E-mail: m.lisagracia@gmail.com.Telephone contact:0034675816681. 


\section{Abstract}

Objective: This study aimed to evaluate the efficiency of treatment of infectious endocarditis (IE) via Selfadministered Outpatient Parenteral Antimicrobial Therapy (S-OPAT) supported by a shortening hospital admission program in a hospitalization-at-home unit (HAH), including a short review of the literature.

Methods: Ambispective cohort study of 57 episodes of IE in 54 patients treated in an HAH unit between 1988 and 2014 who receive S-OPAT after prior intra-hospital clinical stabilization. Characteristics of each episode of IE, safety and efficiency of the care model, were analyzed.

Results: Forty-three (76\%) patients were males with a median age of 61 years $(\mathrm{SD}=16.5)$. A total of $37(65 \%)$ episodes affected the native valve ( $42 \%$ the aortic valve). In $75 \%$, a micro-organism was isolated, of which $88 \%$ were Gram-positive bacteria. No deaths occurred during HAH program, clinical complications appeared in $30 \%$ of episodes, only 6 patients were re-admitted to hospital although no patient died. In the 12 months' follow-up 3 cases had a recurrence. The average cost of a day stay in HAH was $€ 174$ while in traditional cardiology hospitalization was $€ 1100$. The total average cost of treatment of each episode of IE managed entirely in hospital was calculated as $€ 54,723$. Application of the S-OPAT model based on HAH meant a cost reduction of $32.72 \%$.

Conclusions: In suitably selected patients, treatment of IE based on S-OPAT supported by a shortening hospital admission care program by means of referral to a HAH unit is a safe and efficient care model which entails a significant cost saving for the public healthcare system.

Keywords: Efficiency, Hospital-at-Home, Infectious endocarditis, S-OPAT 


\section{Introduction}

Infectious endocarditis (IE) is one of the most serious infectious diseases because of its acute and unpredictable clinical course [1]. Despite the steps forward in diagnosis and treatment [2], its mortality continues to be very high (18\% inpatient and $40 \%$ at one year from follow-up) [3, 4]. Its treatment consumes a large amount of financial resources because of prolonged hospital stay (arising from long term recommendations for parenteral antimicrobial treatment). However, there are hardly any works that evaluate the overall costs of its hospital treatment

Efficiency in the healthcare setting is estimated by means of financial evaluation of costs entailed by comprehensive management of the disease. Cost minimization analysis enables selecting the procedure which, with the same effect, has a lower cost [5]. Therefore, alternative care models to traditional hospitalization that reduce or avoid hospital admission without detriment to a benefit on health should markedly increase efficiency during treatment of hospital processes.

Outpatient Parenteral Antimicrobial Therapy (OPAT), is an alternative care model to traditional hospitalization that has been demonstrated to be effective and safe in many infectious processes [6,7]. Most OPAT programs [8] mainly follow two models. First, infusion in the patient's home with active intervention of nursing personnel called "Healthcare Professional Outpatient Parenteral Antimicrobial Therapy (H-OPAT); and, second, "Selfadministered Outpatient Parenteral Antimicrobial Therapy (S-OPAT)", that is, a model in which the healthcare personnel initially train the patient and/or their caregivers to administer antimicrobials so that healthcare personnel physical presence in the home during infusion is subsequently not necessary. Recently, those of the variant S-OPAT [9] although more limited, are revealing similar efficacy and safety results. In both healthcare processes, the clinician is not too involved in daily follow-up of patients. This is generally limited to monitoring clinical course in the outpatient clinic over a variable period.

In Spain, healthcare expenditure is very high and it is foreseen to increase in the future because of ageing of the population. Sustainability of its healthcare system which is priority in a period of financial crisis such as the one currently will only be possible if we attain maximum efficiency in patient care [10]. Against this backdrop, in our country we have a highly developed system of 150 hospital-at-home units (HAH), in which care devices are managed by internal medicine specialists that provide the patient in their own home diagnosis, care and hospitallike treatments for a limited period just as if they had remained admitted in an acute hospital unit[11].Most HAHs have a wide list of services for the patient including intravenous antibiotic treatment (OPAT) which over recent years has received considerable attention. Other clinical processes addressed by HAHs include pulmonary embolism, acute kidney injury, post-surgery processes as well as decompensation in chronic diseases including chronic obstructive pulmonary disease and congestive heart failure [12-13].HAH has two main treatment 
regimens according to origin of the patients. In the first, the "admissions avoidance scheme", patients come from emergency services, their homes, retirement homes or outpatient clinics and avoid hospital admission. In the second, patients come from hospital wards and seek to shorten usual hospital admission; this is called "early discharge scheme" (when patients remain less than 7 days in hospital) or "shortened hospital admission scheme" (when the patient is admitted for more than 7 days).

The main difference between stand-alone OPAT and HAH is that, in the latter, there is very close clinical follow-up by healthcare professionals. As such, the patient's safety is increased even in complex clinical processes [14].

In the specific case of IE published works on OPAT were anecdotal until a few years ago, due mainly to the reticence to incorporate this complex global process into the portfolio of services for this care modality. However, in the last few years there has been a notable increase in the scientific evidence in this field which coincides with the reiterated support regarding American and European cardiology societies [15-18].

However, there are barely any papers published regarding costs arising from treatment of IE both in hospital and for the OPAT care model. Another gap is that S-OPAT in the context of HAH has not been well-described, and most HAH centers do not utilize S-OPAT. The main aim of this study was to determine the efficiency of treatment of IE using S-OPAT with HAH. The former allows reduced nursing effort by training self- or caregiver administration of antimicrobials, while the latter gives the support of a shortening hospital admission program after an initial period ( $>7$ days) of traditional hospitalization. 


\section{Material and Methods}

\section{Setting}

An ambispective cohort study of patients with IE, diagnosed according to modified Duke criteria [19], and admitted to the HAH unit in a third level University Hospital in Spain was performed from 1998 to 2014.

The methodology to identify IE episodes and essential characteristics were collected in a database devised for the study. The HAH protocol for IE was applied, in addition to evaluation of the efficacy of the HAH program for S-OPAT having previously been reported [20].

IE episodes were diagnosed in hospitalized patients, initially treated in hospital until stabilized, and then transferred to the care of the multidisciplinary HAH team. Following stabilization, consensus decisions were taken regarding the optimum S-OPAT schedules to be conducted in the HAH therapeutic plan, which would include not only IE but also any minor complications that may arise. General criteria for S-OPAT treatment of IE within the HAH program are summarized in Table 1.

The efficiency of S-OPAT was first estimated by calculating the cost per day of stay in the HAH by means of the sum of direct costs (personnel costs, pharmacy consumption, healthcare material, maintenance, amortization of equipment, etc.) and costs charged for other hospital services and structures. We need to point out certain details to understand the cost calculation made. The HAH unit has a care capacity of 56 patients/day and has a physical infrastructure located in hospital. The staff is made up of seven doctors, 17 nurses, seven clinical assistants and two administrative assistants. There are 16 automobiles financed by means of renting to undertake their care work. The healthcare and non-healthcare material together with the pharmacy are supplied by the hospital. Costs are calculated by means of evaluation of direct cost (staff, pharmacy consumption, healthcare material, maintenance, amortization of equipment, etc.) for the unit during 2014; to which we must add the costs charged for other hospital services and structures.

And second, calculating the cost of a day stay in a traditional hospital by means of financial analysis of the 37 patients with IE (25 with entire intrahospital care and 12 with initially intrahospital care and subsequently by means of HAH) who were treated in our hospital and survived during the final years of the study (2013-2014) with the purpose that the financial data obtained are as reliable as possible. Of the 37 patients analyzed individually, they were included in two different groups; an initial control group of 25 patients that received antibiotic treatment entirely in the hospital; the second case group comprised of the 12 patients who initially received intrahospital treatment followed by treatment in HAH. For each patient, the process and complications presented during the IE episode were evaluated coding for the diagnosis-related group (DRG) generated at discharge. The costs assigned to each DRG were calculated by the hospital's analytical accounting unit using the full cost or total attribution system of costs for the care activity administered. Finally, the comparative analysis 
of average cost of a patient who was treated entirely in hospital compared to the patient who received combined treatment, was performed.

\section{Data analysis}

Data were introduced into a database created with the program Microsoft Excel and variables were analyzed by means of the statistical packages SPSS version 11.5 (SPSS Inc. Headquarters, Wacker Drive, Chicago, Illinois, USA).A descriptive analysis was performed expressing quantitative variables in the form of mean \pm standard deviation (SD) and qualitative variables in the form of percentage and proportions.

\section{Ethical Statement}

This study was approved with code 2008.4 by the Ethics Committee of the Autonomous Community of Cantabria, Spain. There was compliance with the ethics principles set out in the Declaration of Helsinki and international Good Clinical Practice ethical regulations. All patients signed informed consent to take part in the study. 


\section{Results}

A total of 57 episodes of EI in 54 patients were included in the study;23 episodes were collected from the retrospective period (1998-2007) and 34 were collated prospectively (2008-2014). This is equivalent to $9.1 \%$ of the total of 624 IE episodes treated by the infectious disease unit of our hospital over that period.

Patients baseline characteristics (basic epidemiological variables, associated comorbidities and history of heart valve disease) and the specific features of the IE episodes are summarized in Table $\mathbf{2}$.

The S-OPAT program showed high efficacy as shown by the results summarized in Table 3. No patient died at home. Clinical complications appeared in approximately $30 \%$ of episodes. Six patients had to return to hospital none of whom died in hospital and two of them returned to the HAH unit to complete their S-OPAT. During the one year follow upto the initial episode, two patients presented a relapse of IE: one of them on two occasions, both requiring surgery because intravenous antibiotic treatment failed. In the other patient, the relapse responded favorably to specific antibiotic treatment and did not present subsequent complications. The results of our current series of 57 episodes of IE and those of other series published that applied different OPAT regimens are summarized in Table 4.

Costs are calculated by means of evaluation of direct cost (staff, pharmacy consumption, healthcare material, maintenance, amortization of equipment, etc.) for the unit during 2014; to which we must add the costs charged for other hospital services and structures (see Table 5). The cost of one day stay in HAH is calculated considering the care data for the HAH unit during 2014 that treated 1,567 admissions which generated a total of 20,480 stays. The overall cost of the unit was $€ 3,569,519$, whereby the average cost of the stay turned out to be $€ 174.29$. During the estimate of traditional hospitalization 15 different DRGs were used to evaluate cost arising from treating 37 patients chosen for this purpose. Therefore, weighting for the stays attributed to each DRG generated by the processes involved (1,562 stays) and quantifying the price/stay for each one of them, an average cost of $€ 1166.80$ per day stay was determined.

For the comparative analysis of costs between treatment of IE according to the traditional regimen (only hospital) and combined treatment model (initially in hospital, subsequently the HAH unit), we need to ascertain the average stay of both groups of patients. In the case of the control group 25 patients received comprehensive treatment on a hospital ward and their average stay was 49 days. Average stay was also 49 days for the case group of 12 patients that received combined treatment (30 days in hospital and 19 days in the HAH unit). With these data, once the cost/stay of the two models and the number of stays to apply in each one of them are identified, the final results are those shown in Table 6. That is, the average total cost of treating an episode of IE in hospital was 54,723.22 euros whilst the cost of doing so by applying both care models in a combined fashion 
was $36,815.58$ euros. From this, we deduce that the combined treatment model saves 17,907.64 euros per episode of IE, $32.72 \%$ in comparison to the conventional treatment model. 


\section{Discussion}

This study shows the efficiency of treatment of patients with IE by means of S-OPAT in comparison with a traditional hospitalization model.

The OPAT model has undergone modifications during our study's broad period in accordance with the onset of new antimicrobials and scientific evidence that led to modifications in traditional antimicrobial recommendations for different infectious processes [21]

Treatment at home by means of various care modalities of infections with traditional criteria for hospitalization is now consolidated with excellent results regarding efficacy, reducing hospital stays, reducing costs of the process and proving to be satisfactory for the patient [22,23]. In the case of IE, because this is a complex infectious process, it was incorporated into treatment under this care model at a late stage, whereby there is little prior experience in this regard $[20,24,25]$. Nonetheless, the repeated support shown both by the American Heart Association (AHA) [17,18] and the European Society of Cardiology (ESC) $[15,16]$ in the last few years, including OPAT in its clinical guidelines as a care modality for IE in selected patients, has led to an increased publication of works, thereby guaranteeing this care option [26-31].

To date, in most published series on OPAT for IE the H-OPAT model predominated; S-OPAT was clearly the minority option. In our previous work [20] we observe that, because the characteristics of patients and episodes of IE were very similar to previous series, the efficacy of the S-OPAT program was excellent because no patient died and the rate of unexpected returns to hospital and relapse was similar to that of other recently published series[28,31]. Moreover, the paper we present here extends the previously published seriesby 9 episodes and is the largest series published in the literature of IE patients who underwent S-OPAT. The results found not only consolidate those obtained previously but they improve them. Our work verifies that the S-OPAT care model is safe and effective to treat IE. Therefore, our care alternative obtains similar healthcare results to the traditional hospitalization model. Furthermore, our data show that between the two care procedures the combined treatment strategy is clearly more efficient than the classical strategy and provides an estimated cost saving of almost $33 \%$.

In a recent review of the OPAT model it was notified that this represents a significant cost reduction in comparison to the traditional hospitalization model but there are still insufficient studies to generalize this statement [32]. In the last few years, papers have been published that try to confirm that this care model, in addition to being effective and safe, is also efficient. Most works are based on the Anglo-Saxon variant of HOPAT and the methodology used for the financial analysis consists of determining the potential saving arising from less day stay cost attributed to the OPAT in comparison to traditional hospitalization [22,33-36].A British OPAT group evaluated 338 acute infectious processes; OPAT turned out to be $41 \%$ cheaper compared to an 
infectious diseases hospital ward [22]. An American group [33] analyzed 398 clinical processes, where OPAT meant a saving for the hospital of \$4 million. Another Canadian study estimated a day cost for OPAT of $14 \%$ compared to traditional hospitalization [34].

Our work provides data on the day stay cost of our HAH but also because it comprehensively lists both direct and indirect costs attributed to our model, a model which has not been comprehensively studied and for which there is very little literature. In this regard, there are no financial evaluation works comparing the different variants of our OPAT. However, the few studies published [35, 36], appear to support the fact that the S-OPAT modality reduces costs more than the H-OPAT model. An American group [35] notified that the cost of the SOPAT represented $10 \%$ of the cost of hospitalization. Subedi S, et al [36] estimated in Australia that the cost of the S-OPAT was $\$ 150$ per day stay whilst in hospital it was $\$ 500-800$. According to all the studies, the reduced price of the process in comparison to H-OPAT would mainly be due to the cost reduction arising from nursing staff given that because it is the patient themself and their caregivers who administer S-OPAT, this leads to a drastic reduction in visits by this staff.

We also estimated a day stay cost of the HAH of $€ 174.29$, very similar to that published in most articles on SOPAT. However, because the HAH is based on the complexity of our S-OPAT model, this is much higher than that of any other variant of the OPAT model, whereby we can state that in this context, our model increases the efficiency of the S-OPAT even further if possible.

In the scope of IE, the little information available on the costs of its treatment in a traditional hospitalization unit is surprising. IE is probably one of the most expensive infectious diseases within the portfolio of services offered by a tertiary level hospital. A French group [37] recently published the financial costs of treating IE, resulting in an average cost of $€ 17,735$ in 2009 , which increased by $34 \%$ during the three years the study lasted. In our study, we estimated an average cost of treating an episode of IE at $€ 54,733.22$, figures well above that published by the French group although we believe our figure is more accurate. Possibly their data are underestimated because the baseline analysis in the DRG assigned exclusively to IE and obviously, this method is incomplete because it does not evaluate each patient individually and we lose data for those complications that occur during the entire clinical course of the disease.

Another French OPAT group [38] published a retrospective study of 39 episodes of IE treated in the hospital, of which 18 were selected for H-OPAT. The study revealed a day stay price of $€ 140$ (not accounting for pharmaceutical costs) H-OPAT; the price of hospital day stay in an infectious disease unit was $€ 1140$, meaning a final saving of $€ 14,850$ per patient. In our series, not only did we obtain higher efficiency given that we saved almost $€ 3000$ more per episode of IE but there was also a higher level of safety. They reported a readmission rate 
of $17.5 \%$ whilst in our case this was $10.5 \%$. These data are of great interest because it confirms that our S-OPAT model is not only more efficient but also safer than the H-OPAT model. The OPAT model in Spain [12, 13] is applied thanks to $\mathrm{HAH}$ units, which provide comprehensive management of the infectious disease at the patient's home, because professionals travel to the home to perform clinical monitoring during the disease. In the Anglo-Saxon model follow-up is performed by the nurse whilst the professional remains at the hospital where the patient comes to be periodically re-evaluated [14].Regular visits at home by the professional guarantee similar clinical outcome to that offered in hospital, thereby increasing the patient's safety by being able to detect the onset of complications early and commence their treatment earlier, thereby avoiding readmission to hospital. Our results have a high degree of internal validity in that the record of data from variables chosen for the study was comprehensive and there was no loss of information. Further, the health-care scheme of the S-OPAT was supported by the HAH team which has had many years of experience in this area. Since its origins in 1984, the administration of intravenous drugs has formed part of the list of services for patients at home. However, this study has some limitations. First, the specific characteristics of our HAH Unit are dependent on a (University) hospital providing advanced care in close collaboration with medical and surgical specialists involved in the management of IE. It can respond rapidly to any clinical incident in the patient's home. All these characteristics make it difficult to extrapolate our conclusions on the safety and efficacy of the health-care model to other health-care contexts. Second, the low number of patients included in our study sample would tend to limit the reproducibility of our scheme in other contexts.

\section{Conclusion}

In summary, IE is a very expensive clinical process; an average of $€ 54,723$ per episode is estimated and the application of two combined care models (initially traditional hospitalization and, subsequently, hospitalizationat-home) clearly increases its efficiency because this means a cost saving of $32.72 \%$ without undermining safety and effectiveness.

\section{Funding}

This paper was sponsored by Merck Sharp \& Dohme (Spain). The sponsor had no role in study design, data collection, data analysis, data interpretation or writing the report.

\section{Declaration of Interests}


The authors would like to thank Yolanda Diaz Hoyas for assisting with preparation of the databases. We are also grateful to Jason Willis-Lee MITI for medical writing assistance during preparation of the final manuscript. Medical writing support was paid for by Merck Sharp \& Dohme (Spain). The authors have no other relevant affiliations or financial involvement with any organization or entity with a financial interest in or financial conflict with the subject matter or materials discussed in the manuscript. This includes employment, consultancies, honoraria, stock ownership or options, expert testimony, grants or patents received or pending, or royalties. 


\section{References}

[1] Cahill TJ, Prendergast BD. Infective endocarditis. Lancet 2016; 387: 882-93

[2] Fernández-Hidalgo, N, Tornos Mas, P. Epidemiología de la endocarditis infecciosa en España en los últimos 20 años. Rev Esp Cardiol. 2013; 66:728-33.

[3] Murdoch DR, Corey GR, Hoen B, et al. Clinical presentation, etiology, and outcome of infective endocarditis in the 21st century: the International Collaboration on Endocarditis Prospective Cohort Study. Arch Intern Med $2009 ; 169: 463-73$.

[4] Muñoz P, Kestler M, De Alarcon A, et al. Current Epidemiology and Outcome of Infective Endocarditis: A Multicenter, Prospective, Cohort Study. Medicine 2015; 94(43):e1816.

[5] Salleras Sanmartí L, Navas Alcalá E, and Domínguez García A: Evaluación de la eficiencia de las intervenciones preventivas y de los programas de salud. In Sierra-López A. (eds): Piédrola Gil, Medicina Preventiva y Salud Pública, 11. a ed. Elsevier Másson, 2008 pp. 1217-1230.

[6] Mirón-Rubio M1, González-Ramallo V2, Estrada-Cuxart O3, et al. Intravenous antimicrobial therapy in the hospital-at-home setting: data from the Spanish Outpatient Parenteral Antimicrobial Therapy Registry. Future Microbiol. 2016; 11:375-90

[7] Barr DA, Semple L, Seaton RA. Outpatient parenteral antimicrobial therapy (OPAT) in a teaching hospitalbased practice: a retrospective cohort study describing experience and evolution over 10 years. Int J Antimicrob Agents. 2012; 39:407-13.

[8] Tice AD, Rehm J, Dalovisio J, et al. Practice guidelines for outpatient parenteral antimicrobial therapy. Clin Infect Dis 2004; 38:1651-72.

[9] Matthews PC, Conlon CP, Berend AR, et al. Outpatient parenteral patients to self-administer at home? A retrospective analysis of a large cohort over 13 years. J Antimicrob Chemother 2007; 60:356-62.

[10] Martínez-González, Miguel A. Conceptos de salud pública y estrategias preventivas: Un manual para ciencias de la salud. Madrid: Elsevier; 2013.

[11] Gonzalez Ramallo VJ, Bouza E. Home intravenous antimicrobial therapy. Med Clin (Barc) 2008; 131:2957. 
[12] Mendoza-Ruiz de Zuazu H, Casas Arrate J, De la Maza I, et al. Home Intravenous antibiotic Treatment: a study in 515 patients. Enfermedades Infecc Microbiol Clin 2005; 23:396-401.

[13] Horcajada JP, Garcia L, Benito N, et al. Specialized home care for infectious disease. Experience from 1995 to 2002. Enferm Infecc Microbiol Clin 2007; 25:429-36.

[14] Gonzalez Ramallo VJ, Segado-Soriano A. Twenty-five years of hospital at home in Spain. Med Clin 2006; 126:332-3.

[15] Habib G, Hoen B, Tornos P, et al. Guidelines on the prevention, diagnosis, and treatment of infective endocarditis (new version 2009): the Task Force on the Prevention, Diagnosis, and Treatment of Infective Endocarditis of the European Society of Cardiology (ESC). Endorsed by the European Society of Clinical Microbiology and Infectious Diseases (ESCMID) and the International Society of Chemotherapy (ISC) for Infection and Cancer. Eur Heart $J 2009$ Oct; 30:2369-413.

[16] Habib G, Lancellotti P, Antunes MJ, et al. Eur 2015 ESC Guidelines for the management of infective endocarditis: The Task Force for the Management of Infective Endocarditis of the European Society of Cardiology (ESC). Endorsed by: European Association for Cardio-Thoracic Surgery (EACTS), the European Association of Nuclear Medicine (EANM). Eur Heart J2015; 36: 3075-128.

[17] Baddour LM, Wilson WR, Bayer AS, et al. Infective endocarditis: diagnosis, antimicrobial therapy, and management of complications: a statement for healthcare professionals from the Committee on Rheumatic Fever, Endocarditis, and Kawasaki Disease, Council on Cardiovascular Disease in the Young, and the Councils on Clinical Cardiology, Stroke, and Cardiovascular Surgery and Anesthesia, American Heart Association: endorsed by the Infectious Diseases Society of America. Circulation 2005; 111:e394-434.

[18] Baddour LM, Wilson WR, Bayer AS, et al. Infective endocarditis: diagnosis, antimicrobial therapy, and management of complications: a statement for healthcare professionals from the Committee on Rheumatic Fever, Endocarditis, and Kawasaki Disease, Council on Cardiovascular Disease in the Young, and the Councils on Clinical Cardiology, Stroke, and Cardiovascular Surgery and Anesthesia, American Heart Association: endorsed by the Infectious Diseases Society of America. Circulation 2015; 132:1435-86.

[19] Li JS, Sexton DJ, Mick N, et al. Proposed modifications to the Duke criteria for the diagnosis of infective endocarditis. Clin Infect Dis 2000; 30:633-638. 
[20] Pajarón M, Fernández-Miera MF, Allende I, et al; Hospital Valdecilla Endocarditis Study Group. Selfadministered outpatient parenteral antimicrobial therapy (S-OPAT) for infective endocarditis: a safe and effective model. Eur J Intern Med 2015; 26:131-6

[21] Esposito, S., Noviello, S., Boccia, G., de Simone, G., Pagliano, P., de Caro, F. Changing modalities of outpatient parenteral antimicrobial therapy use over time in Italy: A comparison of two time periods. Infez. Med. 4, 2, 2016, 137-139

[22] Chapman AL, Dixon S, Andrews D, et al. Clinical efficacy and cost-effectiveness of outpatient parenteral antibiotic therapy (OPAT): a UK perspective. J Antimicrob Chemother 2009; 64:1316-24

[23] MacKenzie M, Rae N, Nathwani D. Outcomes from global adult outpatient parenteral antimicrobial therapy programmes: a review of the last decade. Int Jantimicrob Agents 2014 Jan; 43:7-16

[24] AndrewsMM, von Reyn CF. Patient selection criteria and management guidelines for outpatient parenteral antibiotic therapy for native valve infective endocarditis. Clin Infect Dis 2001; 33:203-9.

[25] Tice AD. Safety of outpatient parenteral antimicrobial therapy for endocarditis. Clin Infect Dis 2002; $34: 419-20$.

[26] McMahon JH, O’Keeffe JM, Grayson ML. Is hospital-in-the-home (HITH) treatment of bacterial endocarditis safe and effective? Scand J Infect Dis 2008; 40:40-3

[27] Larioza J, Heung L, Girard A, et al. Management of infective endocarditis in outpatients: clinical experience with outpatient parenteral antibiotic therapy. South Med J 2009; 6:575-9.

[28] Amodeo MR. Outpatient intravenous treatment for infective endocarditis: safety, effectiveness and one-year outcomes. J Infect 2009; 59:387-93.

[29] Cervera C, Del Rio A, Garcia L, et al. Efficacy and safety of outpatient parenteral antibiotic therapy for infective endocarditis: a ten-year prospective study. Enferm Infecc Microbiol Clin 2011; 29:587-92.

[30] Partridge DG, O’Brien E, Chapman AL. Outpatient parenteral antibiotic therapy for infective endocarditis: a review of 4 years' experience at a UK centre. Postgrad Med J 2012; 88:377-81.

[31] Duncan CJ, Barr D, Ho A, et al. Risk factor for failure of outpatient parenteral therapy (OPAT) in infective endocarditis. J Antimicrob Chemother 2013; 68:1650-4. 
[32] Paladino JA, Poretz D. Outpatient parenteral antimicrobial therapy today. Clin Infect Dis 2010; 51:198-208.

[33] Lai A, Tran T, Nguyen HM, et al.Outpatient parenteral antimicrobial therapy at large Veterans Administration medical center. Am J Manag Care 2013; 19:317- 24.

[34] Wai AO, Frighetto L, Marra CA, et al. Cost analysis of an adult outpatient parenteral antibiotic therapy (OPAT) programme. A Canadian teaching hospital and Ministry of Health perspective. Pharmacoeconomics $2000 ; 18: 451-457$

[35] Ruth CA, Parameswaran GI, Wojciechowski AL, et al. Outcomes and pharmacoeconomic Analysis of a Home Intravenous Antibiotic Infusion Program in Veterans. Clin Ther 2015; 37:2527-35.

[36] Subedi S, Looke DF, McDougall DA, et al. Supervised self-administration of outpatient parenteral antibiotic therapy: a report from a large tertiary hospital in Australia. Int J Infect Dis 2015; 30:161-5.

[37] Sunder S, Grammatico-Guillon L, Baron S, et al. Clinical and economic outcomes of infective endocarditis. Infect Dis (Lond) 2015; 47:80-7.

[38] Lacroix A, Revest M, Patrat-Delon S, et al. Outpatient parenteral antimicrobial therapy for infective endocarditis: a cost-effective strategy. Med Mal Infect. 2014; 44:327-30. 
Table 1. Inclusion criteria for an episode of IE in the HAH shortening hospital admission program of the HUMV

General:

Voluntary participation by the patient and carer(s) after being informed about the

functioning of the $\mathrm{HAH}$

Requirement for a $24 \mathrm{~h} /$ day carer in the home of the patient

HAH operating within the catchment area of $15 \mathrm{~km}$ from the hospital

Specific:

Commitment on the part of the patients and carer(s) to the S-OPAT scheme

Clinical stability (no fever, hemodynamic stability, no cardiac insufficiency, no focal neurological disease

Laboratory criteria (normal leucocyte distribution, decreasing C-reactive protein levels and stable renal function

Absence of atrial-ventricle blockage confirmed using an electrocardiogram

Echocardiographic assessment to confirm decreased size of vegetation and/or absence of para-valvular complications following at least 1 week with suitable antibiotics

HAH: hospitalization-at-home; HUMV: Hospital Universitario Marqués de Valdecilla; IE: Infective endocarditis; S-OPAT: self-administered at home outpatient parenteral antimicrobial therapy 
Table 2. Basal characteristics and specific features of the IE episodes

\section{Basal characteristics}

Age (mean,SD)

$61(16.5)$

Males (n, \%)

43(75.9)

Existence of comorbidity (n, \%)

47(83.3)

Charlson index (mean, SD)

$2.6(2.4)$

Hypertension (n, \%)

26(45.6)

Diabetes (n, \%)

11(19.2)

Non-valvular chronic heart failure (n, \%)

$8(14.5)$

Chronic Obstructive Pulmonary Disease (n, \%)

$6(10.4)$

Chronic renal disease (n, \%)

Neoplasia (n, \%)

$13(22.9)$

$9(16.6)$

$5(8.7)$

Chronic liver disease (n, \%)

$32(56.1)$

Prior valve disease operated surgically (n, \%)

$20(33.3)$

Previous infectious endocarditis (n, \%)

4(7.4)

Specific features of the IE

Type of valve affected (n, \%)

Native

Prosthesis

Location of the valve affected (n, \%)

Mitral

Aortic

Mitro-aortic

Pulmonary

Tricuspid

Site of acquisition (n, \%)

Community

Nosocomial/Healthcare Relations.

Need for heart surgery (n, \%)

Documented principal micro-organism (n, \%)

Staphylococcus aureus

$\mathrm{CNS}^{2}$

Viridans group Streptococci

Streptococcus bovis

Enterococci

Antibiotic most commonly used (n, \%) **

Ceftriaxone

Vancomycin

Ampicillin

Penicillin $G$

Linezolid

Teicoplanin

Cloxacillin

Duration of treatment (weeks, SD)

Intrahospital

$\mathrm{HAH}$

CNS: Coagulase negative staphylococci; COPD: Chronic Obstructive Pulmonary Disease; HAH: hospitalizationat-home IE: infectious endocarditis; SD: standard deviation

*Other documented micro-organisms: Streptococci pneumonia (1, 2,3\%), Abiotrophia defectivus (1, 2.3\%), Aggregatibacter aphrophilus (1, 2.3\%), Escherichia coli (1, 2.3\%), Haemophilus parainfluenzae (1, 2.3\%),

Listeria monocytogenes (1, 2.3\%), Providenza rettgeri (1, 2.3\%), Salmonella typhimurium (1, 2.3\%).

** Other Antibiotics used: cefazolin, ertapenem, gentamycin, meropenem 
Table 3. Efficacy of the HAH program for S-OPAT during IE

Characteristics

$\mathrm{n}(\%)$

Mortality (n, \%)

Major complications requiring unexpected return (n, \%)

Anaphylaxis secondary to allopurinol

Moderate hypopotassaemia

Hepatorrenal syndrome

Multifactorial renal failure

AV blockade requiring pacemaker

Respiratory sepsis requiring ITU

Complications resolved at home (n, \%)

4 episodes of congestive heart failure

3 episodes of catheter-related infection

4 episodes of acute drug reactions

-Vancomycin red man syndrome

-Vancomycin drug fever

-Abnormal liver function tests to rifampicin

-Acute kidney injury because of aminoglucosides

Relapse (n, \%)

$3(5.2)$

HAH: hospitalization-at-home; IE: Infective endocarditis; ITU: intensive therapy unit; S-OPAT: selfadministered at home outpatient parenteral antimicrobial therapy 
Table 4. Outcome of IE in different OPAT series

\begin{tabular}{|c|c|c|c|c|c|c|c|}
\hline $\begin{array}{l}\text { 1st Author } \\
\text { Reference }\end{array}$ & $\begin{array}{c}\text { Pajaron } \\
\text { Current-2016 }\end{array}$ & $\begin{array}{l}\text { McMahon } \\
\text { SJID-2007 }\end{array}$ & $\begin{array}{c}\text { Larioza } \\
\text { SMJ-2009 }\end{array}$ & $\begin{array}{l}\text { Amodeo } \\
\text { JOI-2009 }\end{array}$ & $\begin{array}{c}\text { Cervera } \\
\text { EIMC-2011 }\end{array}$ & $\begin{array}{l}\text { Partridge } \\
\text { PMJ-2012 }\end{array}$ & $\begin{array}{c}\text { Duncan } \\
\text { JAC-2013 }\end{array}$ \\
\hline Type OPAT & S-OPAT & H-OPAT & H-OPAT & $\begin{array}{c}\text { H-OPAT } \\
75 \% \\
\text { S-OPAT } \\
25 \%\end{array}$ & $\begin{array}{l}\text { H-OPAT } \\
\text { S-OPAT } \\
\text { partially }\end{array}$ & $\begin{array}{c}\text { S-OPAT } \\
60 \% \\
\text { Day } \\
\text { Hospital } \\
40 \%\end{array}$ & OPAT \\
\hline IE Episodes & 57 & 40 & 43 & 100 & 73 & 36 & 80 \\
\hline \multicolumn{8}{|l|}{ Outcome } \\
\hline Mortality (\%) & 0 & 0 & 0 & 0 & 4 & 2.7 & 1.2 \\
\hline $\begin{array}{l}\text { Unexpected } \\
\text { returns (\%) }\end{array}$ & 10.5 & 7.5 & 23 & 10 & 16 & 11 & 26 \\
\hline Relapse (\%) & 5.2 & & & 5 & & 2.7 & 8.7 \\
\hline
\end{tabular}

H-OPAT: healthcare outpatient parenteral antimicrobial therapy; IE: Infective endocarditis; OPAT: Outpatient Parenteral Antimicrobial Therapy; S-OPAT: self-administered at home outpatient parenteral antimicrobial therapy 
Operating Costs

Costs charged from other services

Day stay cost
Professionals

713,154

Personnel Costs

Nursing

$1,030,173$

Auxiliary assistants

223,691

Administrative assistants

TOTAL

57,526

$\mathbf{2 , 0 2 4 , 5 4 4}$

Pharmaceutical products

540,596

Other Healthcare Material

90,462

Blood bank

16,100

108,849

67,805

18,237

6142

848,191

696,784

Total Costs

$\mathbf{3 , 5 6 9 , 5 1 9}$

174.29 
Table 6. Cost estimate for the traditional care model and combined mode for the treatment of IE.

\begin{tabular}{lcccc}
\hline Care model & $\begin{array}{c}\text { Average stays } \\
\text { (days) }\end{array}$ & $\begin{array}{c}\text { Unitary average } \\
\text { cost (Euros) }\end{array}$ & $\begin{array}{c}\text { Cost } \\
\text { IE episode (Euros) }\end{array}$ & Total cost (Euros) \\
\hline Hospital alone & 49 & 1116.80 & $54,723.22$ & $\mathbf{5 4 , 7 2 3 . 2 2}$ \\
$\begin{array}{l}\text { Hospital plus } \\
\text { HAH }\end{array}$ & 30 plus 19 days & 174.29 & $33,504.02$ plus $3,311.57$ & $\mathbf{3 6 , 8 1 5 . 5 8}$ \\
& & & &
\end{tabular}

